Research, Society and Development, v. 9, n. 8, e632986124, 2020

(CC BY 4.0) | ISSN 2525-3409 | DOI: http://dx.doi.org/10.33448/rsd-v9i8.6124

\title{
Aprendizagem significativa na educação matemática: o uso do Diagrama CBN
}

Significant learning in mathematical education: the use of the CBN Diagram

Aprendizaje significativo en educación matemática: el uso del Diagrama CBN

Recebido: 29/06/2020 | Revisado: 02/07/2020 | Aceito: 08/07/2020 | Publicado: 22/07/2020

\section{Roger Santos Ferreira}

ORCID: https://orcid.org/0000-0002-6993-9353

Instituto Federal de Educação, Ciência e Tecnologia de Minas Gerais, Brasil

E-mail: roger.ferreira@ifmg.edu.br

Manoel Pereira Junior

ORCID: https://orcid.org/0000-0001-6202-6618 Instituto Federal de Educação, Ciência e Tecnologia de Minas Gerais, Brasil

E-mail: manoel.pereira@ifmg.edu.br

Gláucia do Carmo Xavier

ORCID: https://orcid.org/0000-0003-3133-7354

Instituto Federal de Educação, Ciência e Tecnologia de Minas Gerais, Brasil

E-mail: glaucia.xavier@ifmg.edu.br

\section{Resumo}

Este artigo tem por objetivo analisar e refletir o processo ensino e aprendizagem da área de Educação Matemática e apresentar um produto educacional, voltado para o Ensino Médio, como inovação metodológica. O produto educacional desenvolvido foi o Diagrama CBN, o qual pode ser compreendido como um diagrama de conversões entre bases numéricas. A pesquisa se justificou, uma vez que os livros didáticos, comumente utilizados para o ensino de conversões, oferecem variadas técnicas consideradas complexas por alunos recém-chegados no Ensino Médio Técnico Integrado. A pesquisa foi desenvolvida no Instituto Federal de Minas Gerais, em duas turmas distintas de um dos campi da instituição, em que o Diagrama CBN foi utilizado apenas em uma delas, objetivando um estudo comparativo. A escolha metodológica foi pela técnica da observação participante. A motivação da proposta se baseia no questionamento de práticas tradicionais do ensino em relação à Educação Matemática. Os resultados indicam concretização de aprendizagem significativa, de Ausubel, quando o diagrama é aplicado junto à teoria de conversões entre as bases de sistemas numéricos, melhorando o desempenho acadêmico dos alunos. 
Palavras-chave: Ensino e aprendizagem; Conversão de bases numéricas; Diagrama CBN; Educação matemática, Aprendizagem significativa.

\begin{abstract}
This article aims to analyze and reflect the teaching and learning process in the area of Mathematical Education and present an educational product, aimed at high school, as a methodological innovation. The educational product developed was the CBN Diagram, which can be understood as a diagram of conversions between numerical bases. The research was justified, since the textbooks, commonly used for teaching conversions, offer various techniques considered complex by students recently arrived in integrated technical high school. The research was developed at the Federal Institute of Minas Gerais, in two different classes on one of the institution's campuses, in which the CBN Diagram was used only in one of them for a comparative study. The methodological choice was based on the participant observation technique. The motivation of the proposal is based on the questioning of traditional teaching practices in relation to mathematical education. The results indicate the achievement of significant learning, by Ausubel, when the diagram is applied together with the theory of conversions between the bases of numerical systems, improving students' academic performance.
\end{abstract}

Keywords: Teaching and learning; Conversion of numerical bases; CBN Diagram; Math education; Meaningful learning.

\title{
Resumen
}

Este artículo tiene como objetivo analizar y reflejar el proceso de enseñanza y aprendizaje en el área de Educación Matemática y presentar un producto educativo, dirigido a la escuela secundaria, como una innovación metodológica. El producto educativo desarrollado fue el Diagrama CBN, que puede entenderse como un diagrama de conversiones entre bases numéricas. La investigación se justificó, ya que los libros de texto, comúnmente utilizados para enseñar conversiones, ofrecen diversas técnicas consideradas complejas por los estudiantes que llegaron recientemente a la escuela secundaria técnica integrada. La investigación se desarrolló en el Instituto Federal de Minas Gerais, en dos clases diferentes en uno de los campus de la institución, en la que el Diagrama CBN se usó solo en uno de ellos para un estudio comparativo. La elección metodológica se basó en la técnica de observación participante. La motivación de la propuesta se basa en el cuestionamiento de las prácticas docentes tradicionales en relación con la Educación Matemática. Los resultados indican el 
logro de un aprendizaje significativo, por parte de Ausubel, cuando el diagrama se aplica junto con la teoría de las conversiones entre las bases de los sistemas numéricos, mejorando el rendimiento académico de los estudiantes.

Palabras clave: Enseñando y aprendiendo; Conversión de bases numéricas; Diagrama CBN; Educación matemática; Aprendizaje significativo.

\section{Introdução}

Baseado na tecnologia, da qual a sociedade depende intrinsecamente nos dias atuais, a programação de computadores e manuseio da tecnologia da informação requerem o básico conhecimento de vários sistemas numéricos, os quais são usados para nomear ou representar grandezas numéricas (Tocci, Widmer, \& Moss, 2011). Vale dizer que existem variados sistemas numéricos, dentre os quais se destacam o decimal, o binário, o octal e o hexadecimal, usados em várias áreas relacionadas à tecnologia e Educação Matemática, como eletrônica, informática, computação, mecatrônica, automação, entre outras.

Considerado como o sistema numérico de maior relevância, o sistema decimal, ou de base 10, é composto de dez dígitos ou algarismos, sendo eles: 0, 1, 2, 3, 4, 5, 6, 7, 8 e 9. Por não ser conveniente para o uso em sistemas digitais, o sistema numérico binário, ou de base 2 , é extensivamente utilizado, pois a representação entre apenas dois níveis de tensão elétrica é mais simples de se representar e trabalhar. No sistema binário, apenas os numerais 0 e 1 são utilizados, sendo possível representar qualquer quantidade apenas com esses dois símbolos (Tocci et al., 2011).

Entre cada par origem-destino dos sistemas numéricos, existem técnicas diferentes de conversão, o que se mostra de difícil e lento aprendizado por alunos iniciantes no estudo de disciplinas técnicas, na Educação Básica. Somado a isso, vários são os livros-texto adotados no processo de ensino e aprendizagem desse conteúdo, como por exemplo Floyd (2007), Pedroni (2008), Idoeta e Capuano (2008), além de Tocci et al., (2011). Embora tais livros ofereçam exemplos de conversão usando essas técnicas, a utilização prática acaba gerando dúvidas, uma vez que a conversão, entre todas as quatro bases citadas é realizada por um total de doze técnicas com sutis diferenças. Essas diferenças tênues corroboram para que as técnicas comumente utilizadas sejam consideradas difíceis ou confusas pelos alunos.

Sendo a conversão entre sistemas numéricos um conteúdo aprendido, normalmente, via memorização pelos alunos, surge a questão: um diagrama de conversões de fácil interpretação melhoraria potencialmente o processo de ensino e aprendizagem dessa temática? 
Assim, o objetivo deste trabalho é refletir sobre a prática pedagógica no ensino de conversão de bases numéricas, de forma a produzir significados dentro e fora da escola, por meio de uma reorganização do pensamento matemático. Objetivou-se também apresentar um produto educacional desenvolvido sob forma de um diagrama que organize de maneira sistêmica as conversões entre bases de sistemas numéricos, bem como avaliar experimentalmente a eficiência de sua aplicação em um estudo de caso.

A abordagem metodológica escolhida como principal foi a pesquisa qualitativa, com uso pontual de descrição estatística. Assim, como o objetivo do presente trabalho está mais relacionado ao processo de ensino de conversões entre bases numéricas e menos aos resultados ou dados quantitativos, acredita-se ser a abordagem qualitativa a mais completa, sem deixar de lado, a breve análise quantitativa ocorrida na comparação dos dados obtidos. Como técnica de pesquisa, utilizou-se a observação participante, já que nesse método o agir é observado diretamente no fazer e não constituído por relatos de outros.

O estudo foi desenvolvido com alunos do Ensino Médio Técnico Integrado em um campus do Instituto Federal de Minas Gerais. Contou com a participação de 59 alunos de duas turmas diferentes do mesmo ano. O diagrama $\mathrm{CBN}$ foi utilizado por apenas uma turma, enquanto a outra obteve uma instrução tradicional. Em seguida foi aplicada uma avaliação para comparar o entendimento de ambas.

Este trabalho está organizado em cinco seções. A primeira seção é esta Introdução. A segunda oferece os pressupostos teóricos da aprendizagem significativa como objetivo do processo de ensino e aprendizagem, além dos conhecimentos básicos sobre representação numérica e os tipos de conversões mais utilizadas. A seção três apresenta o Diagrama CBN proposto, suas bases teóricas e sua contribuição como nova tecnologia para a sala de aula visando ao ensino de conversões entre sistemas numéricos. Em seguida, na quarta parte do artigo, tem-se a metodologia utilizada, com a demonstração do experimento realizado. A seção cinco apresenta as análises dos dados obtidos, e, por fim, o último tópico do texto apresenta as considerações finais da pesquisa e as contribuições do Diagrama CBN, como um instrumento relevante e uma estratégia facilitadora para a aprendizagem de conversões de bases numéricas.

\section{O Processo de Ensino e Aprendizagem de Conversões Numéricas}

O processo de ensino e aprendizagem é matéria de vasta pesquisa e variadas teorias no meio acadêmico. Uma vez que o ensino é construído pelas respostas naturais às exigências do 
processo de aprendizagem, mais importante é que o professor acompanhe a aprendizagem do aluno do que se concentre demasiadamente no conteúdo ministrado (Santos, 2001). A aprendizagem do aluno é o foco do ensino e saber ensinar é tão importante para o professor quanto dominar o conteúdo ensinado.

Ausubel, Novak e Hanessian (1980), estudiosos da educação e proponentes da aprendizagem significativa (AS), a qual disserta sobre como os conhecimentos prévios de um aluno se mesclam de forma coerente e interagem de forma plena com ideias expressas simbolicamente (palavras, imagens e operações) defende que para a aprendizagem significativa ocorrer, deve-se estar atento aos conhecimentos prévios dos alunos e ao material utilizado pelo professor que precisa ser potencialmente relevante.

$\mathrm{Na} \mathrm{AS}$ as "ideias expressas simbolicamente interagem de maneira substantiva e nãoarbitrária com aquilo que o aprendiz já sabe” (Moreira, 2011, p.13). Vale dizer que o termo "não-arbitrária" diz respeito a conhecimentos relevantes, ou seja, que já existem na estrutura cognitiva do aluno. Assim, a AS só pode ocorrer a partir de um conhecimento prévio. É preciso se ter uma base para que novos conhecimentos se fixem na base já existente. Já a aprendizagem mecânica, muito comum nas escolas, refere-se à aprendizagem ligada puramente à memorização, conhecida, por docentes e discentes, como "decoreba". Muitas vezes essa aprendizagem ocorre de forma desconectada de um contexto, com objetivo de obter boas notas em avaliações. Com isso, muitas vezes, os alunos fazem uso de uma técnica memorística e, tão logo o conteúdo seja substituído por outro, é esquecido. E assim, o processo se repete, de conteúdo fragmentado em conteúdo fragmentado, aprendizes e ensinantes utilizam a aprendizagem mecânica como uma regra escolar. Porém, vale ressaltar que a aprendizagem mecânica não se apresenta como inverso do conceito de AS.

Ausubel et al. (1980) afirma que elas não constituem uma dicotomia, uma vez que a AS implica compreensão, capacidade de explicar o que sabe e incorporação substantiva de um dado conhecimento, ela pode ocorrer também por meio da memorização. No entanto esse processo não é natural ou automático. Ele só ocorre se houver a existência do que Ausubel et al. (1980) chama de subsunçores adequados, quer dizer, conhecimentos prévios e mais, essa transição de uma aprendizagem mecânica para a significativa depende também da predisposição do aluno em aprender (que não se refere a desejo, mas a um conhecimento primário necessário à aprendizagem de outro conhecimento mais aprofundado), de materiais significativos e mediação do professor.

Como se observa, a aprendizagem precisa ser significativa para ser compreendida, transferida e capaz de dar ao sujeito capacidade de enfrentar novas situações e, chegar até a 
uma aprendizagem sólida, demanda um processo que não combina com a aprendizagem mecânica. Este trabalho objetiva analisar o processo de uma AS por meio de um ensino potencialmente significativo, utilizando para tanto de um diagrama como estratégia e instrumento facilitador.

Em um paralelo ao produto educacional deste trabalho, neste caso, representado pelo Diagrama CBN, as propostas elencadas por Ausubel et al. (1980) se aplicam na seguinte forma:

a) a atribuição de conceitos primitivos como divisão inteira, resto da divisão, potenciação e operações básicas aritméticas, quando aplicadas em algoritmos específicos (sequência de passos lógicos, finita e não dúbia) oferecem significado e, portanto, podem gerar o conhecimento necessário ao entendimento das conversões entre bases de sistemas numéricos, ou seja, o conhecimento prévio sendo necessário e utilizado para novas aprendizagens;

b) ainda que sugerido pelo autor o problema do decora - usa - esquece, causado pela aprendizagem mecânica, a AS de um conteúdo extenso e maçante também se mostra exaustiva para o estudante, podendo ocasionar seu esquecimento após seu uso. Para isso, é imprescindível um instrumento didático que auxilie a mudança de postura do docente, com o intuito de facilitar a aprendizagem significativa dos alunos (Moreira, 2011);

Essa pesquisa buscou novas formas de aprender e, baseada nos estudos de Ausubel et al. (1980), desde a década de 60, acredita na necessidade de se sair do círculo viciante do quadro de giz e apresentações em slides, memorização nas vésperas de provas, findando no esquecimento rápido. $\mathrm{O}$ intuito foi desenvolver um instrumento educacional capaz de facilitar a aprendizagem, de forma que ela se consolide. Como se sabe, partindo do pressuposto cognitivo, um aluno só aprende a partir do que ele já sabe. Assim, as próximas subseções serão destinadas a apresentar um campo de conhecimentos necessários para que haja a predisposição no aprendizado de conversões numéricas.

A seção seguinte é parte dos estudos teóricos do trabalho. Ela apresentará saberes e conhecimentos sobre os sistemas numéricos, também objeto de estudo desta pesquisa. 


\subsection{Sistemas numéricos}

Embora utilizemos letras e palavras em computadores e dispositivos eletrônicos, esses dispositivos não os entendem como os humanos. Para uma máquina, todas essas letras, ou, como definido na computação, esses "caracteres", precisam necessariamente ser convertidos para números (Malvino \& Brown, 1999). Apesar dos seres humanos terem fixado como padrão para seu uso o sistema numérico decimal (DEC), tornando-o confortável nas operações cotidianas, esse não é o único sistema numérico existente. $\mathrm{O}$ estudo e entendimento de outros sistemas numéricos como o binário (base 2 - BIN), o octal (base 8 - OCT) e o hexadecimal (base 16 - HEX) oferece uma compreensão abrangente sobre como os sistemas numéricos funcionam em geral e qual o papel dos algarismos para a Educação Matemática.

É preciso entender os algarismos em um número antes de se discutir as conversões entre os sistemas numéricos. Malvino e Brown (1999) explicam sobre o fundamento de algarismos, utilizando para tanto da metáfora de um hodômetro, o qual inicia-se em 0000, depois de um quilômetro, passa para 0001, depois para 0002 e assim por diante. Dessa forma, os números presentes em cada roda do hodômetro são chamados dígitos, os quais no exemplo do sistema numérico decimal vão sendo incrementados até que chegam ao seu limite superior, ocorrendo o reinício e enviando uma unidade para a casa à esquerda.

O mesmo exemplo do hodômetro aplicado ao sistema binário seria algo com apenas duas rodas: 00. Assim, ao se andar um quilômetro, o resultado seria 01. No segundo quilômetro, 10. No terceiro quilômetro, 11. E no quarto quilômetro? O que ocorre é a conjugação de mais um hodômetro à esquerda do previamente existente, formando: 0100 . Neste caso, reinicia-se o hodômetro da direita, enviando-se uma unidade ao da esquerda.

Sobre a representação numérica, é de comum acordo na literatura se usar o número representativo do sistema numérico para representar sua base, colocando-o de forma subscrita logo à direita do número, conforme apresentado na Tabela 1. Essa diferenciação auxilia a apresentação de números que existem em variadas bases, como $23_{10}, 23_{8}$ e $23_{16}$, o que não ocorre no sistema numérico binário. Isso porque qualquer número de qualquer sistema numérico (arábico) pode ser escrito na forma genérica da Notação Posicional (NP) ou Sistema de Valor Posicional (Tocci et al., 2011; Gaonkar, 1984).

Por exemplo, o número 906 na base decimal é composto por 9 centenas +0 dezenas + 6 unidades, o que em Notação Posicional seria $9 \times 10^{2}+0 \times 10^{1}+6 \times 10^{0}$. Logo, a representação de sistemas numéricos segue a Notação Posicional, denotada segundo a Equação 1, em que: $a=$ dígito, $b=$ base numérica e $n=$ posição: 


$$
\text { Número }=a_{n} \times b^{n}+a_{n-1} \times b^{n-1}+\cdots+a_{1} \times b^{1}+a_{0} \times b^{0}
$$

Tabela 1: Notação Posicional do número 906 em diferentes sistemas numéricos.

\begin{tabular}{|c|c|c|}
\hline $\begin{array}{c}\text { Sistema } \\
\text { numérico }\end{array}$ & Número & Notação Posicional $=\sum_{0}^{n} a_{n} \times b^{n}$ \\
\hline Decimal & $906_{10}$ & $9 \times 10^{2}+0 \times 10^{1}+6 \times 10^{0}$ \\
\hline Binário & $1110001010_{2}$ & $1 \times 2^{9}+1 \times 2^{8}+1 \times 2^{7}+0 \times 2^{6}+0 \times 2^{5}+0 \times 2^{4}+1 \times 2^{3}+0 \times 2^{2}+1 \times 2^{1}+0 \times 2^{0}$ \\
\hline Octal & $1612_{8}$ & $1 \times 8^{3}+6 \times 8^{2}+1 \times 8^{1}+2 \times 8^{0}$ \\
\hline Hexadecimal & $38 \mathrm{~A}_{16}$ & $3 \times 16^{2}+8 \times 16^{1}+\mathrm{A} \times 16^{0}$ \\
\hline
\end{tabular}

Fonte: Elaborada pelos autores.

Observa-se que a posição ocupada por um dígito no número equivale ao seu valor componente no número como um todo. Logo, o índice de sua posição é o expoente de potenciação do sistema numérico no qual se deseja representar um número. Dessa notação, infere-se, portanto, que existe uma relação entre os algarismos, em que um é mais significativo que o outro em sua composição final, sendo que para números inteiros, o dígito à esquerda é o mais significativo (do inglês, "Most Significant Digit" - MSD) e o dígito à direita é o menos significativo (do inglês, “Less Significant Digit”) (Idoeta \& Capuano, 2008).

Considerado menos complicado que o sistema numérico decimal, pois utiliza apenas dois dígitos (0 e 1), o sistema binário também é representado pela Notação Posicional, porém sua base é sempre o número 2 (Floyd, 2007). Vale ressaltar que em sistemas digitais, cada dígito recebe um novo nome: bit. Assim como os demais sistemas numéricos, o bit mais à esquerda é o mais significante (do inglês, "Most Significant Bit" - MSB) e o mais à direita o menos significante (do inglês, "Less Significant Bit" - LSB), ou seja, o bit de menor peso na soma final da composição do número (Pedroni, 2008). Portanto, operações aritméticas básicas como soma, subtração, multiplicação e divisão podem ser realizadas com muito mais simplicidade utilizando-se o sistema numérico binário, uma vez que é composto apenas pelos dígitos 0 e 1.

\subsection{Os sistemas numéricos}

O sistema numérico octal, como indica seu nome, possui oito dígitos, de 0 a 7 , e seu principal uso é na compactação de vetores binários, afinal consegue representar maiores grandezas numéricas com menos dígitos (Pedroni, 2008). Segundo Floyd (2007) e Idoeta e 
Capuano (2008), o sistema numérico octal tem pouca utilização prática na atualidade de sistemas digitais, atuando principalmente como um sistema intermediário entre o binário e o hexadecimal.

Para contextualizar o processo de contagem sequencial, uma comparação entre os sistemas numéricos decimal, binário, octal e hexadecimal é apresentada na Tabela 2. A contagem do sistema octal é próxima à contagem no sistema decimal, porém sem utilizar os dígitos 8 e 9 .

Tabela 2: Exemplo de contagem em diferentes sistemas numéricos.

\begin{tabular}{|c|c|c|c|c|c|c|c|c|c|c|c|c|c|c|c|}
\hline Decimal & 1 & 2 & 3 & 4 & 5 & 6 & 7 & 8 & 9 & 10 & 11 & 12 & 13 & 14 & 15 \\
\hline Binário & 1 & 01 & 11 & 100 & 101 & 110 & 111 & 1000 & 1001 & 1010 & 1011 & 1100 & 1101 & 1110 & 1111 \\
\hline Octal & 1 & 2 & 3 & 4 & 5 & 6 & 7 & 10 & 11 & 12 & 13 & 14 & 15 & 16 & 17 \\
\hline Hexadecimal & 1 & 2 & 3 & 4 & 5 & 6 & 7 & 8 & 9 & $\mathrm{~A}$ & $\mathrm{~B}$ & $\mathrm{C}$ & $\mathrm{D}$ & $\mathrm{E}$ & $\mathrm{F}$ \\
\hline
\end{tabular}

Fonte: Elaborada pelos autores.

Mais usado na área da computação, o sistema numérico hexadecimal possui 16 dígitos: 0, 1, 2, 3, 4, 5, 6, 7, 8, 9, A, B, C, D, E e F (Idoeta \& Capuano, 2008). Diferentemente dos demais sistemas numéricos apresentados, observa-se que a letra A representa o dígito A, que por sua vez representa a quantidade numérica 10. Por se tratar de um sistema alfanumérico também de notação posicional, esse sistema consegue representar grandes valores numéricos com poucos dígitos, se mostrando ideal para o armazenamento e transmissão de grandes valores.

Uma vez que cada sistema numérico tem suas particularidades, vantagens e desvantagens de uso, faz-se necessário que existam técnicas e métodos (algoritmos) para se realizar de forma eficaz a conversão entre esses sistemas. De todos os quatro sistemas numéricos abordados até aqui, existem simples técnicas de conversão entre cada um deles, com exceção apenas do caso de conversões entre os sistemas octal e hexadecimal, os quais necessitam de uma conversão intermediária, normalmente para o binário, mas que também pode ocorrer pelo sistema decimal.

Há, portanto, conversões diretas e bidirecionais entre BIN $\Leftrightarrow$ OCT, BIN $\Leftrightarrow$ DEC, BIN $\Leftrightarrow$ HEX, DEC $\Leftrightarrow$ OCT e DEC $\Leftrightarrow$ HEX. Indiretamente, de forma simples, existem as conversões entre OCT $\Leftrightarrow$ HEX, usando para tanto do sistema binário ou decimal como intermediário. A próxima seção apresentará com mais detalhes cada uma dessas conversões. 


\subsection{Conversão DEC $\rightarrow$ (BIN ou OCT ou HEX)}

A técnica de conversão do sistema numérico decimal para as demais bases segue uma mesma linha de raciocínio, utilizando-se da técnica de sucessivas divisões pela base de destino (Tocci et al., 2011), conforme exemplificado na Figura 1.

A Figura 1 apresenta exemplos de conversão do sistema decimal para as demais bases, sendo (a) a base binária, (b) a base octal e, (c) a base hexadecimal. A técnica de conversão nesse caso sugere que se façam sucessivas divisões inteiras do número de origem em base decimal pela base de destino. Salienta-se o fato de que na divisão inteira observa-se o valor de resto a cada iteração da etapa de divisões, resultando assim valores que comporão o resultado final da conversão, concatenados com o quociente (resultado) da última divisão inteira (Idoeta \& Capuano, 2008).

Figura 1: Exemplos de conversão por sucessivas divisões .

(a) DEC $\rightarrow$ BIN, (b) DEC $\rightarrow$ OCT, (c) DEC $\rightarrow$ HEX.

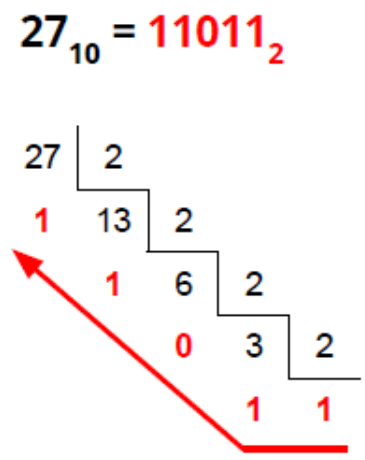

(a)

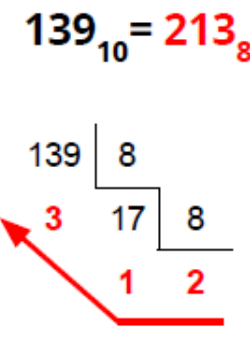

(b)
$1262_{10}=4 E_{16}$

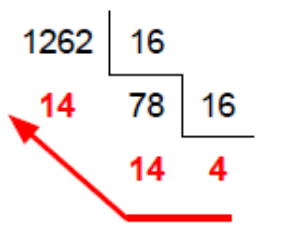

(c)

Fonte: Elaborada pelos autores.

No caso da Figura 1 (a), conversão para a base binária, observa-se que os restos das divisões por dois sempre serão 0 s ou $1 \mathrm{~s}$, obtendo-se, portanto, o resultado final no sentido da seta de indicação na Figura 1, a qual representa que o bit mais significativo deve ser o último quociente e o bit menos significativo deve ser o resto da primeira das divisões (Floyd, 2007; Idoeta \& Capuano, 2008).

Para os demais casos, (b) e (c) da Figura 1, o procedimento é o mesmo, alterando-se apenas o número divisor pelo número representante da base de destino. Para o caso da conversão DEC $\rightarrow$ HEX é observável a ocorrência de restos que ultrapassam o numeral 9, 
sendo, portanto, necessária a tradução dos restos entre 10 e 15 para as respectivas letras do sistema numérico hexadecimal, conforme demonstrado na Figura 1 (c).

No sentido inverso de conversão apresentada acima, a técnica de conversão que possui o sistema numérico decimal como destino da conversão de todas as demais bases utiliza-se da Notação Posicional, apresentada na seção 2.2 para realizar sua tarefa. A Figura 2 demonstra a aplicação de todos os três casos.

Como apresentado na Figura 2, o cálculo do somatório dos números de origem utilizando a Notação Posicional com a base igual ao sistema numérico de origem da conversão, resulta em seu valor na base decimal, tornando assim a utilização da Notação Posicional a técnica padrão de conversão de qualquer sistema numérico para o sistema decimal (Tocci et al., 2011).

Figura 2: Exemplos de conversão por Notação Posicional.

(a) BIN $\rightarrow$ DEC, (b) OCT $\rightarrow$ DEC, (c) HEX $\rightarrow$ DEC.

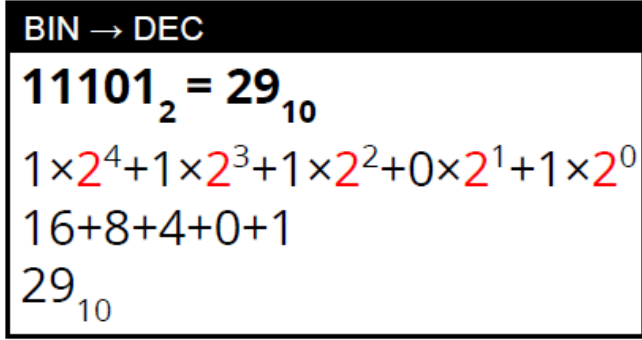

(a)

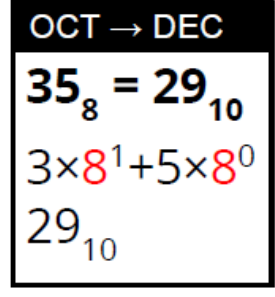

(b)

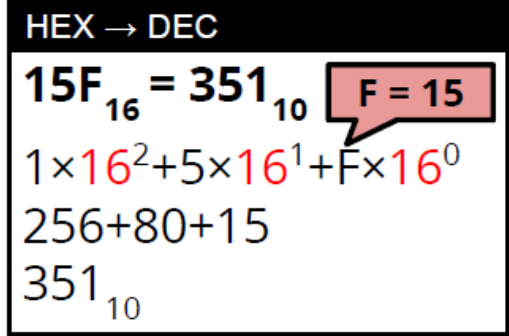

(c)

Fonte: Elaborada pelos autores.

Ademais, o sistema numérico octal pode ser representado por apenas três bits, pois o número $111_{2}$ representa o número $710 \mathrm{e}$, esse, por sua vez, é o número de limite superior no sistema octal. Assim, a Tabela 3 é inferida é deve ser consultada para se realizar de forma facilitada as conversões entre esses dois sistemas numéricos. 
Tabela 3: Equivalências entre os dois sistemas - BIN e OCT.

\begin{tabular}{|c|c|}
\hline OCTAL & BINÁRIO \\
\hline 0 & 000 \\
\hline 1 & 001 \\
\hline 2 & 010 \\
\hline 3 & 011 \\
\hline 4 & 100 \\
\hline 5 & 101 \\
\hline 6 & 110 \\
\hline 7 & 111 \\
\hline
\end{tabular}

Fonte: Elaborada pelos autores.

A conversão no sentido BIN $\rightarrow$ OCT é baseada no agrupamento de dígitos em grupos de três dígitos e posterior substituição pelo valor observado na Tabela 3 (Floyd, 2007; Idoeta \& Capuano, 2008). A Figura 3 apresenta um exemplo dessa técnica de conversão.

Figura 3: Exemplo de conversão BIN $\rightarrow$ OCT.

$$
1001101_{2}=115_{8}
$$

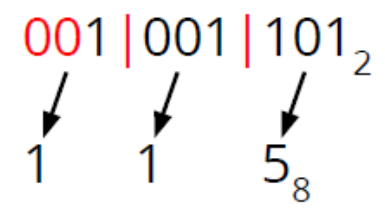

Fonte: Elaborada pelos autores.

Observando-se o agrupamento de dígitos do bit menos significativo para o mais significativo, ressalta-se a necessidade de se completar os bits do grupo mais à esquerda (MSB) com zeros para que o mesmo também seja compatível com os valores da Tabela 3, conforme demonstrado no exemplo. Dessa forma, a conversão no sentido inverso, OCT $\rightarrow$ BIN funciona exatamente de forma inversa à conversão apresentada de BIN $\rightarrow$ OCT. Para tanto, basta que cada dígito do sistema octal de origem seja escrito como um pequeno grupo de três bits, preservando-se a ordem dessa simples tradução. A Figura 4 apresenta um exemplo de tal técnica de conversão. 
Figura 4: Exemplo de conversão OCT $\rightarrow$ BIN.
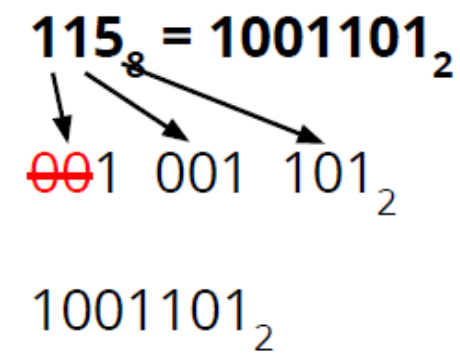

Fonte: Elaborada pelos autores.

Conforme demonstrado pela Figura 4, a conversão OCT $\rightarrow$ BIN é a simples tradução de cada dígito para seu equivalente em três bits no sistema binário (Idoeta \& Capuano, 2008).

O que se observa é que todas as regras de conversão aplicadas entre os sistemas binário e octal se aplicam igualmente em substituição para o sistema hexadecimal, contudo, para esse caso a tabela de grupos de bits é formada por quatro bits, quantidade essa necessária e suficiente para representar todos os números do sistema numérico hexadecimal (Floyd, 2007). A Tabela 4 sumariza as equivalências para a conversão nesse caso.

Tabela 4: Equivalências entre os dois sistemas - BIN e HEX.

\begin{tabular}{|c|c|}
\hline HEXADECIMAL & BINÁRIO \\
\hline 0 & 0000 \\
\hline 1 & 0001 \\
\hline 2 & 0010 \\
\hline 3 & 0011 \\
\hline 4 & 0100 \\
\hline 5 & 0101 \\
\hline 6 & 0110 \\
\hline 7 & 0111 \\
\hline 8 & 1000 \\
\hline 9 & 1001 \\
\hline A & 1010 \\
\hline B & 1011 \\
\hline C & 1100 \\
\hline D & 1101 \\
\hline E & 1110 \\
\hline F & 1111 \\
\hline
\end{tabular}

Fonte: Elaborada pelos autores. 
De forma semelhante à conversão do caso anterior (envolvendo o sistema octal), a conversão de BIN $\rightarrow$ HEX (Figura 5 (a)) precisa agrupar de quatro em quatro bits da direita para a esquerda (LSB para MSB) o número em base binária e, por conseguinte, substituir cada um dos grupos pelo seu respectivo dígito equivalente, conforme apresentado na Tabela 4. Para o sentido inverso de conversão, HEX $\rightarrow$ BIN (Figura 5 (b)), basta que cada dígito do sistema hexadecimal seja traduzido no respectivo grupo de quatro bits, tal qual é exemplificado pela Figura 5, que demonstra exemplos dos dois casos desta seção (Floyd, 2007; Idoeta \& Capuano, 2008).

Conforme abordado anteriormente, não há uma forma simples de se realizar a conversão entre os sistemas numéricos octal e hexadecimal, sendo necessária uma conversão intermediária para o binário ou decimal.

Figura 5: Exemplo de conversão BIN $\rightarrow$ HEX e HEX $\rightarrow$ BIN.

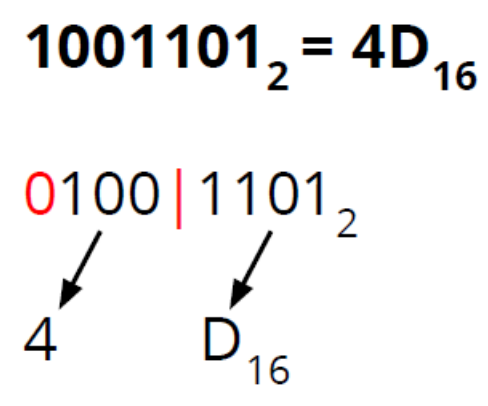

(a)

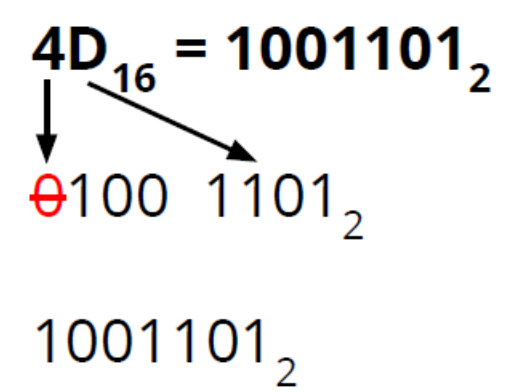

(b)

Fonte: Elaborada pelos autores.

Esta seção 2 apresentou o conteúdo essencial ao entendimento das conversões entre as bases de sistemas numéricos mais utilizados na área técnica. Vale lembrar que esses são conhecimentos necessários para que o aluno consiga transpor o desafio de aprendizado desse conteúdo tão essencial às matérias mais avançadas, porém, devido à expressiva quantidade de detalhes, é necessário um instrumento facilitador no processo de ensino-aprendizagem que possa organizar as ideias (como um mapa mental) e todo o conteúdo exposto até aqui, proposto neste trabalho como o Diagrama CBN, detalhado na seção 3.

\section{Diagrama CBN}

O diagrama apresentado neste estudo se apresenta como um produto educacional voltado ao auxílio do entendimento e compreensão das técnicas de conversão entre os 
sistemas numéricos binário, octal, decimal e hexadecimal. Os livros adotados como bibliografia básica em Projetos Pedagógicos de Cursos voltados às áreas que envolvem eletrônica digital, como Floyd (2007), Idoeta e Capuano (2008) e Tocci et al. (2011) trazem variados capítulos com exemplos e exercícios sobre cada técnica de conversão. Embora seja oferecido um rico conteúdo em tais livros, a sintetização de todas as técnicas de conversão fica a cargo do leitor, o qual nem sempre é capaz de realizar tal simplificação em um modelo mental, levando em conta a complexidade e o nível de abstração do conteúdo apresentado.

O diagrama apresentado na Figura 6, desenvolvido nesta pesquisa, juntamente à explicação de seu uso nos itens a seguir, buscam oferecer o suporte necessário ao ensino e aprendizagem das conversões entre tais sistemas numéricos. Esse diagrama foi criado como uma possibilidade de solução metodológica inovadora.

Figura 6: Diagrama CBN.

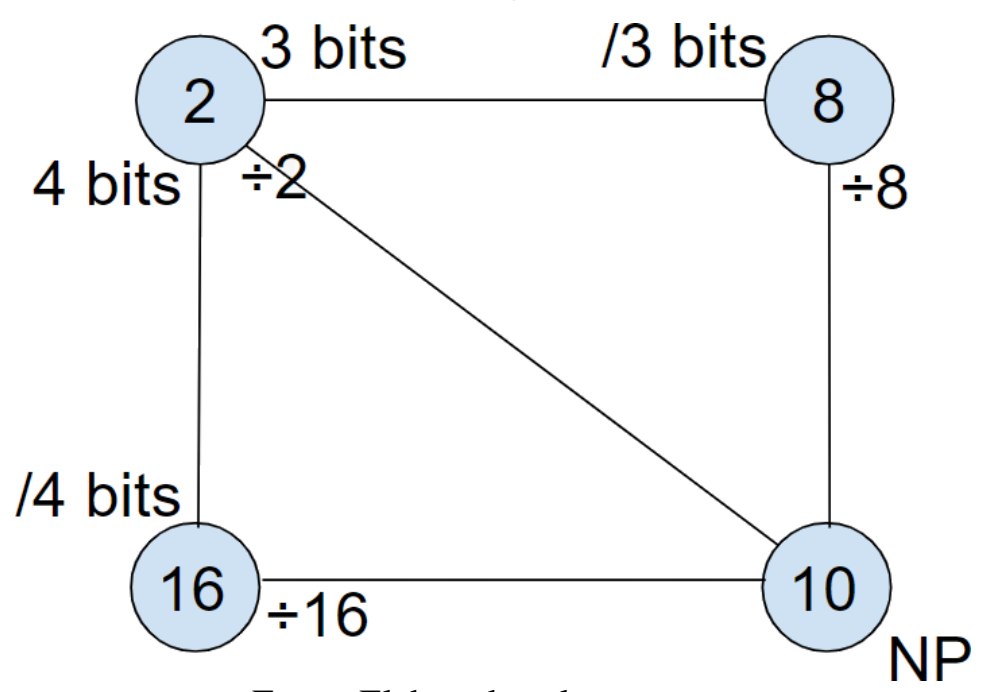

Fonte: Elaborada pelos autores.

Composto por nós, que representam os sistemas numéricos, e arestas, que representam sentidos duplos de conversão, na Figura 6, observam-se os seguintes detalhes do diagrama de conversões proposto:

- NP: na conversão de todos os sistemas BIN, OCT e HEX para o sistema DEC, utiliza-se a Notação Posicional, o que é indicado pela sigla NP ao lado do nó de base 10 .

- $\div \mathbf{n}$ : a conversão de DEC para todos os outros sistemas segue o seguinte procedimento: divide-se o número decimal sucessivamente pela base $\mathbf{n}$ de destino. 
Research, Society and Development, v. 9, n. 8, e632986124, 2020

(CC BY 4.0) | ISSN 2525-3409 | DOI: http://dx.doi.org/10.33448/rsd-v9i8.6124

O resultado é composto pelo último quociente agregado aos restos de cada divisão, lidos da última divisão para a primeira.

- n bits: nas conversões envolvendo BIN, OCT e HEX, cada número de origem deve ser escrito como n bits (completando-se com zeros à esquerda, se necessário), agregando-se os bits resultantes.

- /n bits: divide-se o número de origem em blocos de $\mathbf{n}$ bits da direita para a esquerda e verifica-se o valor de cada grupo correspondente na base de destino.

O ensino desse conteúdo, por vezes considerado confuso por alunos iniciantes nas disciplinas da área de Educação Matemática, como a eletrônica, pode ser adaptado ao uso do Diagrama CBN aqui apresentado, utilizando-se para tanto da Figura 6, juntamente com seu manual (tópicos acima deste parágrafo) e também com as Tabelas 3 e 4.

\section{Metodologia e Avaliação Experimental}

Nesta seção será apresentada a avaliação experimental do Diagrama CBN proposto, bem como a metodologia utilizada, além de serem apresentadas as análises dos dados coletados, a fim de responder à questão problema deste estudo: um diagrama de conversões melhoraria potencialmente o processo de ensino e aprendizagem do conteúdo de conversões entre sistemas numéricos?

Primeiramente, para se avaliar o efetivo ganho ou perda de desempenho no uso do Diagrama CBN, elaborou-se o conteúdo de uma aula de aproximadamente 60 minutos, abrangendo o conhecimento de conversões inteiras entre as diferentes bases de sistemas numéricos, não considerando casos mais específicos, como a conversão de números fracionários. Essa aula foi idealizada para ser ministrada para duas turmas iniciantes em cursos técnicos integrados da área de eletrônica/tecnologia, sendo que foi previamente verificado que os alunos ainda não tinham sido apresentados de maneira formal ao assunto de sistemas numéricos antes dessa aula experimental. Os alunos, participantes da pesquisa, têm idades entre 14 e 17 anos.

Por isso, a técnica escolhida neste estudo foi a observação participante, pois foi levado em conta o envolvimento direto que o investigador tem com um grupo social que estuda. Ademais, esta técnica aproxima o pesquisador do objeto de pesquisa, porém mantendo a distinção. Para isso, a aula foi programada de forma que foram apresentados os conceitos teóricos (grande parte da seção 2 deste trabalho), exemplos de conversões e pequenos 
intervalos de no máximo cinco minutos como desafios para que a turma tentasse realizar dois exemplos de cada conversão. Esse momento objetivou encarar desafios e construir coletivamente o conhecimento científico.

A forma de ministrar o conteúdo baseou-se na ideia de que, para uma das turmas (Turma A), foi apresentado e explicado um dos caminhos no Diagrama CBN, à medida que cada conceito teórico era apresentado. Para a outra (Turma B), não foi apresentado o Diagrama CBN, rotulando-se essa segunda turma como o modelo de ensino tradicional desse conteúdo. Introduziu-se a aula apenas afirmando que se estavam testando duas formas de ensinar um mesmo conteúdo, mas sem detalhes mais explicativos. Ao final da aula, foi entregue um pequeno teste aos alunos, pontuado em 100 pontos e de tempo de resposta de no máximo 60 minutos, para que fosse avaliada a diferença de desempenho entre a Turma A e a Turma B.

Devidamente autorizados pelos pais e/ou responsáveis, que permitiram tal experimento, por meio da assinatura de um Termo de Assentimento Livre e Esclarecido (TALE), participaram do experimento 31 alunos da Turma A e 28 alunos da Turma B.

\section{Análise dos Dados}

As métricas usadas para avaliar os resultados obtidos foram aquelas comumente utilizadas na literatura com avaliações experimentais, como: média, mediana e desvio padrão. A média, mais conhecida como média aritmética, oferece um valor médio de toda a amostra, caracterizando, neste experimento, a nota média da turma. A mediana é o valor que divide a metade inferior, da metade superior de um conjunto numérico. Assim, seu valor neste experimento indica qual a nota mediana da turma, o que pode ser bem diferente da nota média. O desvio padrão foi utilizado para medir o quanto é dispersa a amostra das notas da turma com relação à média, mostrando assim se o desempenho da turma foi mais disperso (heterogêneo) ou se o aproveitamento ficou mais próximo (homogêneo) à média aritmética global.

Uma métrica extra, não quantitativa, observada na realização do experimento foi o engajamento das turmas. Devido à complexidade do conteúdo e o curto tempo de ministração da aula, observou-se que a Turma $\mathrm{B}$, que não tinha o Diagrama $\mathrm{CBN}$, se mostrou um pouco mais perdida, questionando-se como fazia uma ou outra questão que eles tinham acabado de fazer há poucos minutos. Na Turma $\mathrm{A}$, os alunos se voltaram ao Diagrama CBN e conseguiram dirimir as dúvidas. 
Research, Society and Development, v. 9, n. 8, e632986124, 2020

(CC BY 4.0) | ISSN 2525-3409 | DOI: http://dx.doi.org/10.33448/rsd-v9i8.6124

O Gráfico 1 sumariza os resultados obtidos e os cálculos das métricas de avaliação utilizadas. De acordo com o gráfico, observa-se que a média aritmética simples de notas da Turma A (com o Diagrama CBN) se mostrou superior à média de notas da Turma $\mathrm{B}$, o que também é acompanhado de maneira próxima pela medida da diferença entre as medianas obtidas. O gráfico ainda apresenta o desvio padrão reduzido para a Turma A.

Os dados apresentados expressam o resultado esperado pela hipótese deste trabalho: o Diagrama CBN auxilia de forma prática o processo de ensino-aprendizagem sem "decorebas", uma vez que não apresenta de forma explícita os conhecimentos prévios necessários à transposição do desafio oferecido no aprendizado desse conteúdo, mais sim, organiza as ideias necessárias para tal.

Gráfico 1: resultados coletados do experimento (em nota - máximo = 100 pontos).

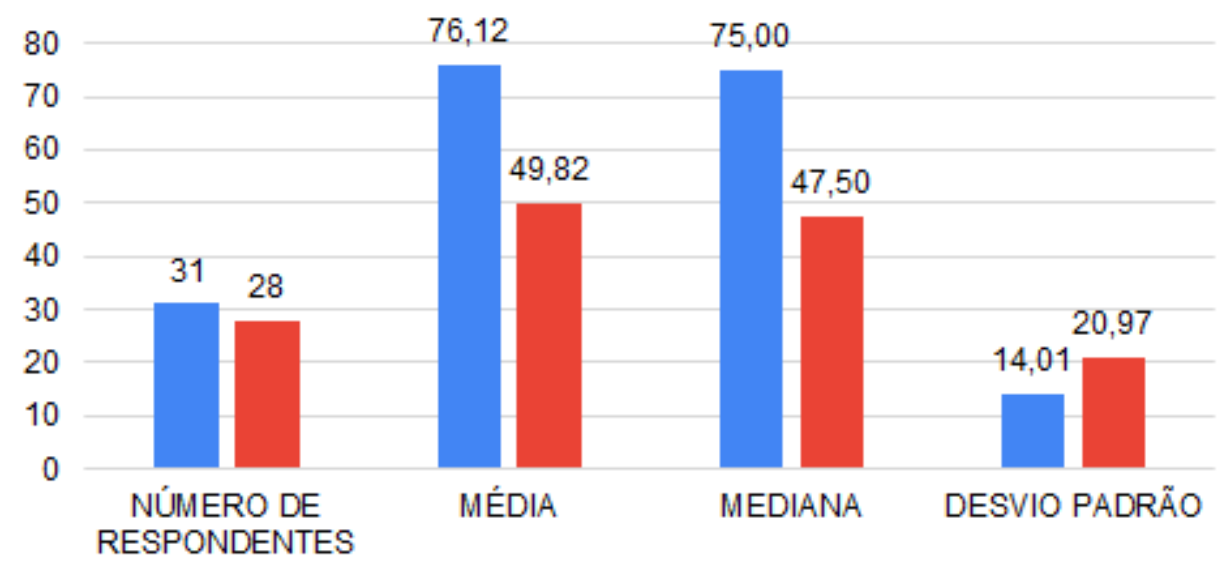

-TURMA A (com diagrama) =TURMA B (sem diagrama)

Fonte: Elaborada pelos autores.

Conforme Ausubel et al. (1980) postula, um material potencialmente relevante é instrumento fundamental para que haja a aprendizagem significativa de discentes. Somado a isso, os pesquisadores deram a atenção devida ao conhecimento prévio desses estudantes. Isso, porque para Ausubel et al. (1980) atribuir significados a novos conhecimentos só ocorre a partir da existência de saberes especificamente relevantes, ou seja, o conhecimento prévio. Vale dizer que a aprendizagem significativa tem como característica principal a interação dos conhecimentos prévios (especificamente relevante) com conhecimentos novos.

Observa-se que o uso do diagrama CBN tem potencial para facilitar o entendimento dos alunos em relação às conversões de bases numéricas. Este fato é corroborado por diversos comentários dos alunos que receberam o diagrama (Turma A), tais como: "usando o 
diagrama não precisei decorar todas as regras de conversão, que me parecem confusas", "com o diagrama foi muito mais fácil fazer os exercícios", "se não fosse o diagrama não daria conta, professor". Por outro lado, a turma que não recebeu o diagrama (Turma B) teve dificuldades, observadas em comentários como: "professor já esqueci tudo!", "não consegui fazer essa conversão aqui!", "professor, como que converte de octal para decimal mesmo?".

Ressalta-se que os conteúdos ministrados nunca tinham sido vistos por ambas as turmas, tendo sido ministrados no dia do experimento, em uma aula de 60 minutos interativa através de exercícios resolvidos em forma de desafio. Esse desafio consistia em dar o resultado final de dois exercícios de conversão explicados naquele mesmo momento, conforme descrito em detalhes na seção 4.

Como foi visto nesta pesquisa, Ausubel et al. (1980) defende a teoria de Aprendizagem Significativa que ocorre,

Quando novos conhecimentos (conceitos, ideias, proposições modelos, fórmulas) passam a significar algo para o aprendiz, quando ele é capaz de explicar situações com suas próprias palavras, quando é capaz de resolver problemas novos, enfim, quando compreende, (Moreira, 2011, p. 60).

Portanto, uma aprendizagem só terá sentido se for significativa e, para tal, demanda, prioritariamente, de ambiente propício, material eficaz, predisposição para aprender e conhecimento prévio.

\section{Resultados e Considerações Finais}

Este trabalho buscou refletir o processo ensino aprendizagem da Educação Matemática e buscou desenvolver um produto educacional na forma de um Diagrama CBN para auxílio ao ensino e aprendizagem de conversões de bases de sistemas numéricos. Foi realizado um levantamento de dados, no qual se avaliou o uso do Diagrama CBN para o ensino de conversões entre bases numéricas em duas turmas distintas do ensino médio integrado, sendo uma delas com o diagrama (Turma A) e a outra sem (Turma B).

Para responder à questão problema deste estudo, foram avaliados (de 0 a 100) 59 testes respondidos pelas duas turmas, resultando em uma melhora na média aritmética simples das notas em aproximadamente 26 pontos para a Turma A (com o uso do Diagrama CBN). Para corroborar o resultado obtido, a Mediana da Turma A também se mostrou acima do valor observado para a Turma B, resultando aproximadamente 27 pontos acima. 
Complementando esse resultado, as notas da Turma A se mostraram mais homogêneas entre a própria turma, comprovado pelo decréscimo de aproximadamente sete pontos no desvio padrão entre as notas. Além dos resultados quantitativos apresentados, observou-se uma melhor orientação dos alunos da Turma A quando se depararam com questões como: Converta o número 1378 para o sistema binário, sabendo como interpretar o caminho no diagrama e lembrando, de fato, de seus conhecimentos previamente obtidos, tais alunos conseguiram distinguir que fazer em seguida. Esse dado demonstra mais equilíbrio entre a aprendizagem dos alunos, melhora no desempenho acadêmico, se comparado com a turma B e, analisando não só os resultados, mas o processo ensino e aprendizagem, os alunos demonstraram menos dúvidas nas atividades de sala e no teste aplicado, o que denota mais segurança diante do conteúdo que, naturalmente, é mais complexo para alunos iniciantes, principalmente no Ensino Médio.

Constatou-se que iniciar o trabalho a partir dos conhecimentos especificamente relevantes, ou seja, prévios (subsunçores), tais como: notação posicional, sistema numérico decimal, divisão inteira, resto da divisão, potenciação e operações básicas aritméticas, foram essenciais para que os alunos se conscientizassem do conhecimento que já tinham sobre algarismos, representação numérica, sistemas numéricos e, consequentemente, conversões. $\mathrm{O}$ Diagrama CBN, desenvolvido para este estudo, foi capaz de demonstrar que, a partir do conhecimento já adquirido anteriormente, eles poderiam acrescentar novos, pois tinham o que precisavam: conhecimento prévio adequado e predisposição para aprender. Ou seja, é necessário partir do ponto em que o aluno se encontra e avançar dali adiante. São os conhecimentos prévios que darão aos alunos a predisposição para aprender, uma vez que não se pode avançar em uma caminhada qualquer pulando etapas grandes.

Portanto, o Diagrama CBN demonstrou ser um instrumento de aprendizagem importante para a AS sobre conversões de bases de sistemas numéricos. O produto educacional criado pode ser considerado um instrumento no auxílio à AS, uma vez que ele é um material de aprendizagem potencialmente significativo.

Conclui-se, portanto, que o Diagrama CBN proposto traz benefícios no processo de ensino e aprendizagem e que seu uso em conjunto a novas posturas educacionais de aulas com menos memorização mecânica pode, de fato, contribuir com o bom desempenho da turma, uma vez que o Diagrama CBN não dificulta o problema a ser resolvido, como ocorre com outras metodologias, mas se ele se torna um guia de qual caminho seguir, dentre tantas opções, o que demonstra a importância de desenvolver produtos educacionais facilitadores da AS. 


\section{Referências}

Ausubel, D. P., Novak, J. D., \& Hanesian, H. (1980). Psicologia educacional. Rio de Janeiro, Interamericana. Tradução para português, de Eva Nick et al., da segunda edição de Educational psychology: a cognitive view

Idoeta, I. V., \& Capuano, F. G. (2008). Elementos de eletrônica digital, 40.ed. Érica Ltda: São Paulo.

Floyd, T. (2007). Sistemas digitais: fundamentos e aplicações, $9^{a}$ ed. Bookman: Porto Alegre.

Gaonkar, R. (1984). Microprocessor architecture, programming and applications with the 8085/8080A, 1.ed. Wiley Eastern Ltda: Nova Delhi.

Malvino, A. P., \& Brown, J. A. (1999) Digital computer electronics, $3^{\text {a }}$ ed. Glencoe/McGrawHill: Nova York.

Moreira, M. A. (2011). Teorias de aprendizagem. 2.ed. EPU: São Paulo.

Pedroni, V. A. (2008). Digital electronics and design with VHDL, 1.ed. Morgan Kaufmann/Elsevier: Burlington.

Santos, S. C. (2001). O processo de ensino-aprendizagem e a relação professor-aluno: aplicação dos "sete princípios para a boa prática na educação de ensino superior". 8(1). Caderno de Pesquisas em Administração: São Paulo.

Tocci, R. J., Widmer, N. S., \& Moss, G. L. (2011). Sistemas digitais: princípios e aplicações. 11. ed. Pearson/Prentice Hall, São Paulo.

\section{Porcentagem de contribuição de cada autor no manuscrito}

$$
\begin{gathered}
\text { Roger Santos Ferreira - 33,33\% } \\
\text { Manoel Pereira Junior - 33,33\% } \\
\text { Gláucia do Carmo Xavier - 33,33\% }
\end{gathered}
$$

